\title{
Brain potentials to emotional pictures are modulated by alexithymia during emotion regulation
}

\author{
Sarah Walker - Daryl B. O'Connor • \\ Alexandre Schaefer
}

Published online: 26 May 2011

(C) Psychonomic Society, Inc. 2011

\begin{abstract}
Alexithymia is a personality trait associated with the reduced ability to regulate, identify, and communicate feelings or emotions and is often linked to psychosomatic disorders. The present study used event-related potentials (ERPs) to investigate the relationship between individual differences in alexithymia and emotion regulation. Participants classified as scoring either high or low on the revised form of the Toronto Alexithymia Scale (TAS-20; Taylor, Bagby, \& Parker Psychother Psychosom 57:34-41, 1992) were asked to view negative and neutral images, adopting three different regulation strategies (expressive suppression, cognitive reappraisal, and attend) while ERPs were recorded. Results revealed an inverse relationship between TAS scores and emotion-related ERP activity during suppression, but not during reappraisal or a control "attend" condition. These results were observed in both early and late ERP latencies. These findings are interpreted according to potential differences between high- and low-TAS individuals regarding the frequency of prior utilization of suppression-based regulation strategies.
\end{abstract}

Keywords Affect - Attention - Alexithymia Event-related potential $\cdot$ Emotion regulation

S. Walker $(\bowtie) \cdot$ D. B. O'Connor

University of Leeds,

Leeds, England, UK

e-mail: s.a.walker88@hotmail.co.uk

A. Schaefer $(\bowtie)$

University of Durham,

Durham, England, UK

e-mail: alexandre.schaefer@durham.ac.uk

\section{Introduction}

Deficits in emotion regulation are thought to play an important role in the onset and maintenance of emotional disorders such as depression and anxiety (Jackson, Malmstadt, Larson, \& Davidson, 2000). The effects of different types of emotion regulation have been examined extensively. These can include acceptance-based strategies (i.e., experience one's emotions fully), emotion suppression (i.e., suppress emotional thoughts), and distraction (Bryant, Moulds, \& Guthrie, 2001; Campbell-Sills, Barlow, Brown, \& Hofmann, 2006a, 2006b; Hayes et al., 2004; Kashdan, Barrios, Forsyth, \& Steger, 2006). A popular approach to emotion regulation suggests that regulation strategies can be categorized into antecedent-focused strategies (e.g., cognitive reappraisal) and response-focused strategies (e.g., expressive suppression) (e.g., Gross, 1998b). Previous research indicates that using reappraisal or suppression can lead to markedly different consequences. For instance, previous research has shown that reappraisal is associated with positive emotional experiences, such as increased life satisfaction (Kashdan et al., 2006), lower symptoms of depression, higher levels of environmental mastery, personal growth, self-acceptance, and a clearer purpose in life (Garnefski, Teerds, Kraaij, Legerstee, \& van den Kommer, 2004; Gross \& John, 2003). Conversely, suppression has been associated with negative emotional experiences, such as higher levels of depressive symptoms and lower self-esteem (Gross \& John, 2003), increases in physiology, including increased eye blinking and sympathetic nervous system activation (Gross \& Levenson, 1993, 1997), and increased distress and negative affect (Ehring, Tuschen-Caffier, Schnulle, Fischer, \& Gross, 2010; Kashdan et al., 2006).

Often neglected within this literature however, is an examination of individual differences. In particular, alexithy- 
mia is a personality dimension that has a strong potential link with emotion regulation. Alexithymia is a trait in which individuals have a reduced ability to regulate, identify, and communicate feelings or emotions (Franz, Schaefer, Schneider, Sitte, \& Bachor, 2004; Sifneos, 1973). Importantly, alexithymia seems to be characterized by an emotion regulation style overreliant on suppression, rather than reappraisal, strategies (e.g., Dimsdale et al., 1986; Franz et al., 2004; Grabe et al., 2004; Kessler, Kammerer, Hoffmann, \& Traue, 2010; Richter et al., 2006; Swart, Kortekaas, \& Aleman, 2009). For instance, Kessler et al. found a positive relationship between TAS scores and individual differences in expressive suppression. Similarly, Swart et al. found significant relationships between alexithymia scores and the Emotion Regulation Questionnaire (ERQ; Gross \& John, 2003). Specifically, they found that high alexithymics were more likely to use suppression than were low alexithymics. Conversely, Swart et al. also found that high alexithymics were less likely to use reappraisal than were low alexithymics, although this effect was smaller than the effect obtained for suppression. A potential consequence of these phenomena is that individuals high and low in alexithymia might have developed different expertise in the utilization of reappraisal and suppression, leading to potential implications for the relative efficiency of different regulation strategies for high and low alexithymics. For instance, if individuals who are low in alexithymia use suppression less frequently than do high alexithymics, they should be less efficient when they attempt to use suppression, because they are less used to this type of strategy. Following Swart et al.'s results, the opposite pattern could be expected for reappraisal: Individuals with high levels of alexithymia should be comparatively inefficient when attempting to use reappraisal, because they are less used to this technique than are low alexithymics.

The goal of this study was therefore to examine whether alexithymia modulates suppression- and reappraisal-based emotion regulation. In order to examine this question, we recorded event-related brain potentials (ERPs) to emotional pictures. ERPs of emotional stimuli provide a reliable index of emotional processing and are known to be sensitive to emotion regulation (e.g., Hajcak, Moser, \& Simons, 2006; Hajcak \& Olvet, 2008; Moser, Most, \& Simons, 2010). In addition, different subdivisions of ERPs to emotional pictures can refer to distinct subcomponents of emotional processing (for a review, see Olofsson, Nordin, Sequeira, \& Polich, 2008). Specifically, early ERPs to emotional pictures $(\sim 100-300 \mathrm{~ms})$, including $\mathrm{P} 1 / \mathrm{N} 1$ and $\mathrm{P} 2 / \mathrm{N} 2$ complexes, are often thought to reflect rapid and probably automatic mobilization of attentional resources by emotional information (Carretié, Hinojosa, Martin-Loeches, Mercado, \& Tapia, 2004; Olofsson et al., 2008; Olofsson \& Polich, 2007; Schupp, Cuthbert, et al., 2004; Schupp, Junghofer, Weike, \& Hamm, 2003), whereas the late positive potential (LPP) to emotional pictures is sensitive to the motivational relevance of stimuli and is thought to reflect an overt, postperceptual analysis of the features of the emotional stimuli/situation in order to facilitate its processing and encoding (Cuthbert, Schupp, Bradley, Birbaumer, \& Lang, 2000; Schupp, Junghofer, Weike, \& Hamm, 2004). Therefore, the morphology and timing of a potential modulation of ERPs to emotion regulation by alexithymia might provide insights into the nature of attentional and cognitive processes where alexithymia and emotion regulation interact. In the present study, alexithymia was measured with the revised Toronto Alexithymia Scale (TAS; Taylor, Ryan, \& Bagby, 1985), the TAS-20 (Bagby, Parker, \& Taylor, 1994; Taylor, Bagby, \& Parker, 1997), one of the most widely used measures of alexithymia (Taylor, 2000). During the experiment, participants were exposed to blocks of negative and neutral pictures. Before each block of pictures, participants were instructed to reappraise, suppress, or simply attend to each image.

\section{Method}

\section{Participants}

Fifty-one healthy students and staff from the University of Leeds (U.K.) were recruited. Participants were excluded if they were left-handed or had a neurological condition. In addition, given the potentially disturbing nature of the negative stimuli used in the study, potentially vulnerable participants were also excluded. These were defined as participants who had a history of psychiatric illness, those taking psychotropic medication, participants who have seen a psychologist/psychiatrist in the past 6 months, and participants who scored above 15 on the Beck Depression Inventory (Beck, Ward, Mendelson, Mock, \& Erbaugh, 1961), since this is the threshold for possible depression in community samples (Beck \& Steer, 1987). Of the 51 participants tested, 5 participants were excluded (3 participants had excessive EEG artifacts, and 2 were behavioral outliers). This left a final sample of 46 to be analyzed. Although much research on alexithymia has relied on a taxometric approach in which participants were typically chosen from low and high ends of alexithymia measures, recent research suggests that alexithymia is better characterized as a dimensional, rather than a taxometric, construct (Ferguson et al., 2009; Ferguson, Wiliams, O'Connor, Bibby, \& O'Carroll, 2008; Parker, Keefer, Taylor, \& Bagby, 2008). Consistent with this approach, we chose to test participants from a broad range of the TAS distribution, instead of considering only the low and high ends, and we analyzed our data using a correlational approach. However, in order to 
also obtain a more standard analysis of our ERP data, we also performed analyses of variance (ANOVAs) comparing individuals scoring high and low on the TAS. Therefore, we defined high-TAS and low-TAS groups on the basis of a TAS cutoff score (48) that allowed us to create two groups that had mean scores similar to those in previous research (Franz, Schaefer, \& Schneider, 2003). ${ }^{1}$ Using this approach, of the 46 participants included, 28 were low TAS (10 males and 18 females; TAS-20 $=36.46)$, and 18 were high TAS ( 7 males and 11 females; TAS-20 $=56.56$ ), with a mean age of 24.56 years $(S D=6.73)$. This approach using both correlational and group-based analyses allowed us to (1) follow the standard of ANOVA-based analysis used in ERP research, (2) retain the dimensional nature of alexithymia to be analyzed via correlation, and (3) ground our operationalization of alexithymia in previous research (Franz et al., 2003). Participants were paid $£ 20$ for their participation. All participants provided informed consent, and the experiment was approved by the University Departmental Ethics Committee.

\section{Stimuli}

Two hundred forty color pictures were chosen from the International Affective Picture System (IAPS; Lang, Bradley, \& Cuthbert, 1999), depicting 120 unpleasant, highly to moderately arousing events (e.g., mutilation, individuals held at gunpoint, images of starvation, car accidents) and 120 neutral events (e.g., household objects, neutral faces), which were medium in valence and low in arousal. In the present study, the IAPS instruction and affective ratings manual was used to assess normative ratings of the images used (Lang et al., 1999). This showed that the two categories (negative and neutral) differed significantly from each other in IAPS normative valence ratings $(M=5.0$ and 2.2 for neutral and unpleasant contents, respectively) and normative arousal ratings $(M=3.2$ and 6.2 for neutral and unpleasant contents, respectively).

\section{Alexithymia}

The TAS-20 (Bagby, Parker and Taylor 1994; Bagby, Taylor and Parker 1994; Taylor et al., 1992) was used to measure the construct alexithymia. Participants were asked to rate each of the 20 statements (e.g., I am able to describe my feelings easily) on a 5-point Likert scale ranging from 1 (strongly disagree) to 5 (strongly agree). The internal consistency of the scale is well established (Taylor et al., 1992). Research has also indicated high levels of convergent, discriminant, and concurrent validity for the scale (Bagby, Taylor, \& Parker, 1994; Zech, Luminet, Rimé, \& Wagner, 1999). In the present study, the scale yielded good internal consistency with an alpha of .90 . In addition to overall alexithymia score, the subdimensions of alexithymia were also explored. These include difficulty identifying feelings (DIF), difficulty describing feelings (DDF) and externally oriented thinking (EOT). The DIF is used to measure individual differences in perceived difficulty in identifying emotions, the DDF is used to measure the perceived difficulty in describing emotions, and the EOT is used to measure the tendency of individuals to focus their attention externally (Bagby, Parker, \& Taylor, 1994; Taylor et al., 1997). Previous research suggests that these subscales are not always highly intercorrelated and may reflect distinct facets of alexithymia (see Gohm \& Clore, 2000; Loas et al., 2001).

\section{Procedure and design}

The experiment consisted of 9 practice and 240 experimental trials divided into three blocks (attend, reappraisal, and suppression). The 240 experimental trials consisted of 120 negative and 120 neutral images from the IAPS (Lang et al., 1999). In each block, there were 80 trials, each with 40 negative and 40 neutral images randomly intermixed. Each block was assigned to a specific regulation strategy (attend, reappraise, or suppress). The order of the reappraise, attend, and suppress blocks was fully counterbalanced across participants. Within each block, there were two 1-min breaks, one halfway through and one at the end.

Participants were initially given written instructions about each regulation task before the experiment (adapted from Gross, 1998a). Next, the researcher discussed the details of each regulation strategy with the participant to ensure full understanding of the task. Moreover, specific regulation instructions were shown again on a screen before each block of trials. For attend blocks, participants were simply asked to focus on the images carefully. In the reappraisal condition, however, participants received the following instructions:

Firstly you will see a cross, please focus on this cross and wait for a picture to appear. It is important that you focus on the picture carefully. Please try and adopt a detached, unemotional and neutral attitude as you view the image. In other words, as you view the image, try to think about what you are seeing objectively, in terms of the technical aspects of the events you observe. Please try to think about what you are seeing in such a way that you don't feel anything at all.

Example 1: If you see an image of an injured individual, try and think about the image in a distant and detached fashion, or even try and imagine the situation improving. In such an instance you may even adopt a medical perspective where you would 
objectively and analytically evaluate the image in a more problem focused manner. In other words, try not to think about the image in a personally-relevant, emotional way.

Example 2: If you see an image where someone is being threatened by a gun or knife, again try and imagine the situation in a distant and detached fashion. You may even imagine the image is a scene from a film, where the individuals are just actors, and therefore no one is in any real danger.

In the suppression condition, participants received the following instructions:

Firstly you will see a cross, please focus on this cross and wait for a picture to appear. It is important that you focus on the picture carefully. If you have any feelings while focusing on the image, please try your best not to let these feelings show. That is try to behave in such a way that a person watching you would not know you were feeling anything.

On each trial, participants were initially presented with a black fixation cross at the center of the screen for 2,000 ms. IAPS pictures were then displayed for $8,000 \mathrm{~ms}$; the order of these pictures was fully randomized within each block. After each picture, participants were shown a screen containing a self-report measure of negative affect using a 7 -point scale $(1=$ weak negative affect and $7=$ strong negative affect). Participants responded to the scale using the keyboard.

Electrophysiological recording, data reduction, and analysis

EEG was recorded with a 128-channel net connected to a high-input amplifier (Electrical Geodesics, Inc., Eugene, OR) at a rate of $500 \mathrm{~Hz}(0.01-$ to $100-\mathrm{Hz}$ bandwidth) and with an impedance of $\leq 20 \mathrm{k} \Omega$. EEG data were recorded using a $\mathrm{Cz}$ reference and were digitally converted to an average mastoids reference. EEG data were analyzed using the ERP module of BESA 5.1 (MEGIS Software GmbH, Gräfelfing, Germany). Following inspection of raw data, bad channels $(<5 \%)$ were replaced using a spherical spline interpolation method implemented in BESA. Data was filtered offline $(0.1-40 \mathrm{~Hz})$, corrected for eye movements (Berg \& Scherg, 1994), segmented into epochs between $200 \mathrm{~ms}$ before (baseline) and 1,500 ms after stimulus onset, and baseline corrected. For each channel, epochs with a difference between the maximum and minimum voltage amplitude of $>120 \mu \mathrm{V}$ and a maximum difference between two adjacent voltage points of $>75 \mu \mathrm{V}$ were rejected (after eye movement artifact correction).

In line with past ERP research using visual emotional stimuli, data analysis focused on anterior, central, and posterior sites, since these are the main sites where emotion effects occur (Herrmann et al., 2008; Junghofer, Bradley, Elbert, \& Lang, 2001; Pastor et al., 2008; Schupp et al., 2000; Schupp, Junghofer, et al., 2004). Electrode data were averaged according to six scalp regions in which data from specific sets of electrodes were pooled together: left anterior, right anterior, left central, right central, left posterior, and right posterior. The left anterior clusters included EGI electrodes 13, 20, 24, 27, 28, and 29; the right anterior clusters included 111, 112, 117, 118, 123, and 124; the left central clusters included $30,35,36,37,42$, and 53; the right central clusters included 80, 86, 87, 93, and 104; the left posterior clusters included 58, 59, 60, 65, 66, and 67; and the right posterior clusters included $84,85,91,92$, 97, and 98. Following a careful visual inspection of our waveforms and a review of the literature on early ERP correlates of emotion (Carretié et al., 2004; Olofsson et al., 2008; Olofsson \& Polich, 2007; Schupp, Junghofer, et al., 2004), mean ERP amplitudes were extracted from four latency windows: 60-100 ms (P1), 90-130 ms (N1), 170$220 \mathrm{~ms}$ (P2), and 220-280 ms (N2). Additionally, the LPP was analyzed in consecutive $200-\mathrm{ms}$ time bins from 400 up to 1,400 ms. ERP waveforms locked to the onset of emotional pictures were created for each of the six experimental conditions: negative-suppression, negative-reappraisal, negative-attend, neutral-suppression, neutral-reappraisal, and neutral-attend.

In order to examine potential relationships between emotion regulation and alexithymia, we first computed a difference score between ERPs to negative and ERPs to neutral pictures in order to obtain an index of the magnitude of emotion-related brain activity. Computing this score was motivated by the fact that the difference between ERPs to emotional and neutral stimuli is widely seen as a reliable index of emotion-related neural activity (e.g., Olofsson et al., 2008) and by the fact that our analyses showed that this difference was, overall, significant in our data (see the Results section). We next computed Pearson correlations testing the relationship between TAS scores and the difference score separately for each regulation condition, each time window, and each electrode cluster. In order to conform to standard ERP statistical analyses, we also computed a 3 (anterior-posterior: frontal vs. central vs. posterior) $\times 2$ (hemisphere: right vs. left) $\times 2$ (image: negative vs. neutral) $\times 3$ (regulation: attend vs. reappraisal vs. suppression) $\times 2$ (TAS: high vs. low) mixed ANOVA for each time window. If differences in TAS scores modulate emotional processing differently according to reappraisal and suppression, negative versus neutral contrasts should have different magnitudes for high and low TAS, depending on whether suppression or reappraisal is being employed. Therefore, we predicted a regulation $\times$ image $\times$ TAS interaction. If this interaction was found in any time window, 
subsidiary analyses focused on TAS $\times$ image interactions separately for each level of regulation and, next, on negative versus neutral contrasts for each TAS group if the subsidiary interaction was significant. Anterior-posterior, hemisphere, regulation, and image were the within-subjects factors, and TAS the between-subjects factor. Greenhouse-Geisser corrections were applied where relevant. For the self-report data, a 2 (image: negative vs. neutral) $\times 3$ (regulation: attend vs. reappraisal vs. suppression) $\times 2$ (TAS: high vs. low) mixed ANOVA was conducted, with image and regulation as the within-subjects factors and TAS as the between-subjects factor.

\section{Results}

\section{Self-report data}

The mixed ANOVA computed on self-reported negative affect revealed a main effect of image, $F(1,45)=195.22, p<.001$, with greater levels of negative affect elicited by negative images, as was expected. A regulation $\times$ image interaction, $F(1,45)=34.86, p<.001$, was also found, reflecting a somewhat reduced effect of image in the reappraisal condition, as compared with the suppression and attend conditions, $F(1,45)=116.63,176.1,213.2$, respectively, all $p$ s $<.001$, consistent with previous research (Gross, 1998a). Similar to previous studies (Newton \& Contrada, 1994; Papciack, Feuerstein, \& Siegel, 1985; Rabavilas, 1987), no reliable differences in self-reported emotional feelings were found between low- and high-TAS individuals (c.f., Table 1). However, an unexpected correlation between TAS scores and differences between self-report scores for emotional and neutral images was found for reappraisal, $r=.39, p<.01$. No significant correlations were found for the suppression or attend conditions.

\section{ERP data}

A visual inspection of our data revealed a typical differentiation between negative and neutral image conditions in both early and late time windows, replicating previous research (Olofsson

Table 1 Mean negative affect ratings for the three regulation tasks in those low and high in TAS (with standard deviations in parentheses)

\begin{tabular}{lll}
\hline & Low TAS & High TAS \\
\hline Attend negative & $4.03(1.48)$ & $4.21(1.29)$ \\
Attend neutral & $1.18(0.22)$ & $1.22(0.21)$ \\
Reappraisal negative & $3.26(1.18)$ & $2.86(1.32)$ \\
Reappraisal neutral & $1.15(0.18)$ & $1.13(0.19)$ \\
Suppress negative & $3.81(1.37)$ & $3.98(1.61)$ \\
Suppress neutral & $1.18(0.25)$ & $1.19(0.21)$ \\
\hline
\end{tabular}

et al., 2008). A closer inspection indicates that this pattern varies between high and low TAS during the suppression condition, in which the differentiation between negative and neutral images is smaller in high-than in low-TAS individuals. This effect seems to be particularly robust in the P2/N2 complex. Both the correlational and ANOVA analyses, explained in more detail below, confirmed these observations.

\section{Correlation analysis}

In order to obtain an index of emotional processing, we computed difference scores between ERPs to negative and neutral images for each regulation condition (attend, reappraisal, and suppression), time window, and scalp region (frontal, central, and posterior). Computing difference scores was possible because the differentiation between negative and neutral waveforms was, overall, significant (see Fig. 1 and ANOVAs below). Each difference score was then correlated with TAS scores. Since we did not find major regional differences (with one exception noted below), we averaged all regional clusters into a whole-scalp cluster. As is shown in Table 2, significant negative correlations between TAS and ERP difference scores were found in both early and late time windows - specifically, during the suppression condition. Although no significant correlations with reappraisal were observed using whole-scalp clusters, we found a positive correlation between TAS and image difference scores in the posterior region during reappraisal in the time window of $1,000-1,200 \mathrm{~ms}, r=.30, p<.05$.

In order to examine the relative contribution of the TAS subscales to the observed main correlations between TAS and suppression difference scores, we ran correlations between the three subscales (DIF, DDF, and EOT) and the ERP difference scores in the suppression condition, using whole-scalp clusters. As is shown in Table 3, the relationship between TAS scores and emotion difference scores in the suppression condition seems to be driven mostly by the DIF subscale.

In order to explore this question further, we tested whether the correlations between the ERP difference scores and DIF were more negative than the correlations between ERP difference scores and DDF and EOT, using the procedure recommended by Meng, Rosenthal, and Rubin (1992). Only time windows where the correlations with DIF were significant were considered. With the exception of EOT in P1 and DDF in N1, the correlations between ERP difference scores and DIF were significantly more negative than the correlations involving DDF and EOT, using a one-tailed significance criterion. The two-tailed significance criterion was exceeded for all comparisons in the $\mathrm{N} 2$ time window and in the difference between DIF and EOT in the 400-600 ms time window.

Overall, these results indicate a negative relationship between alexithymia scores and the ERP differentiation 


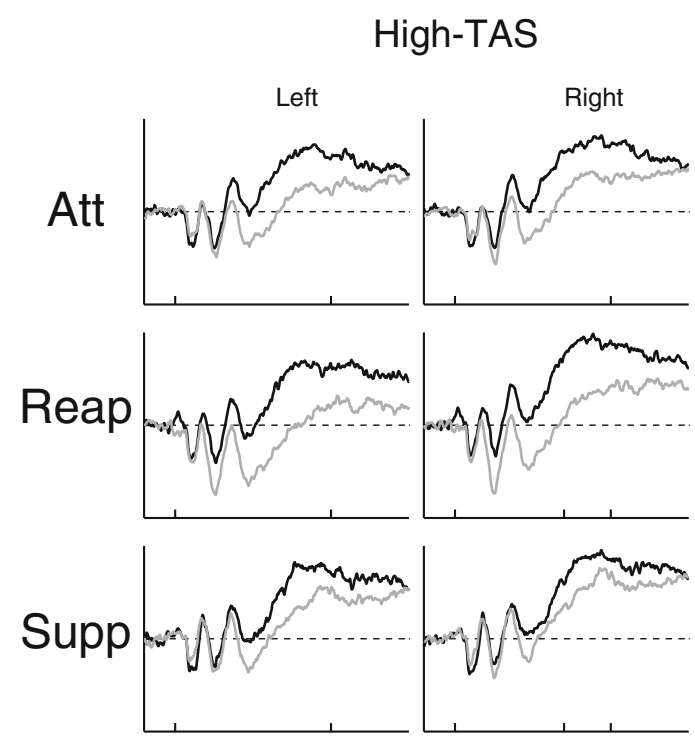

Frontal

Low-TAS
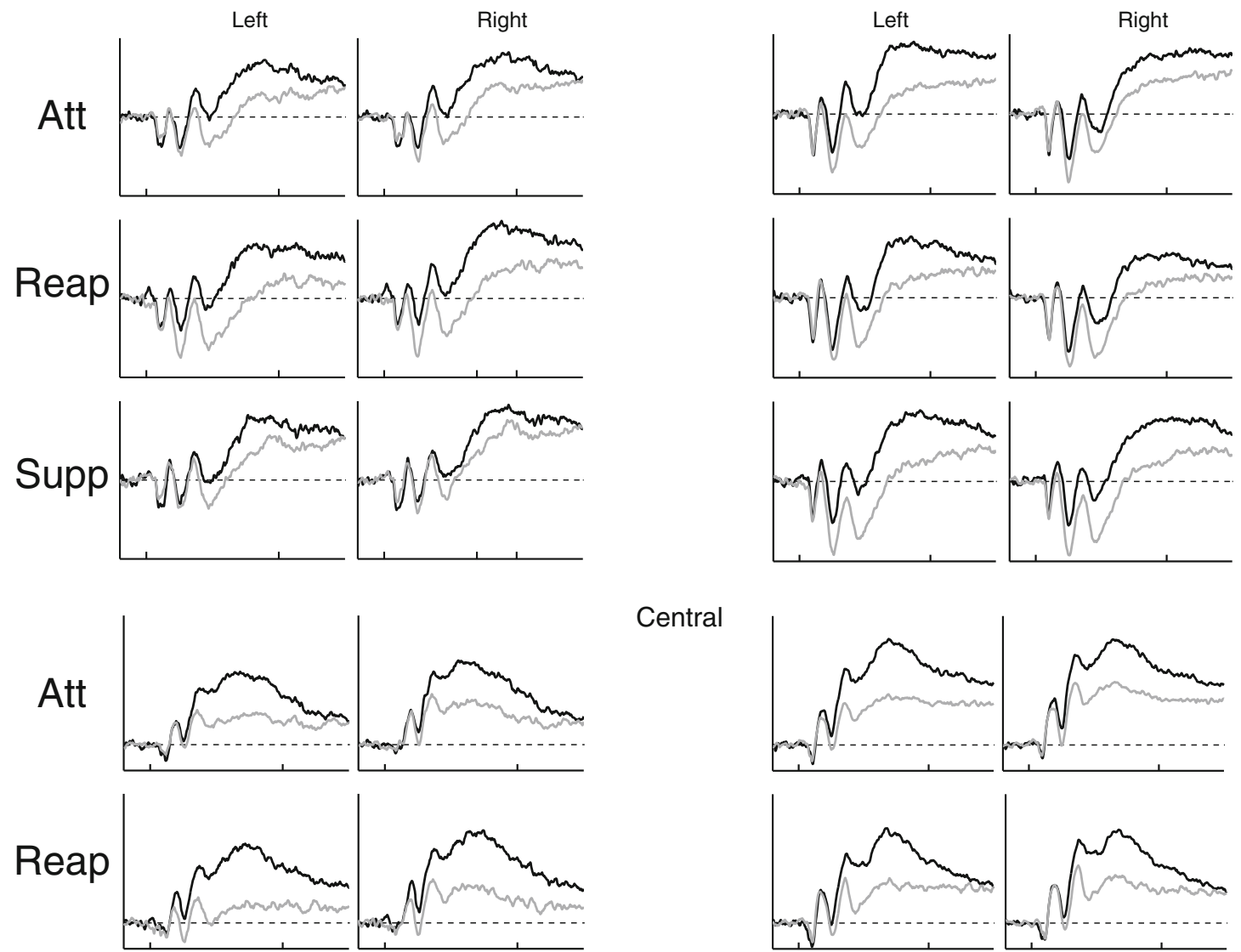

Central
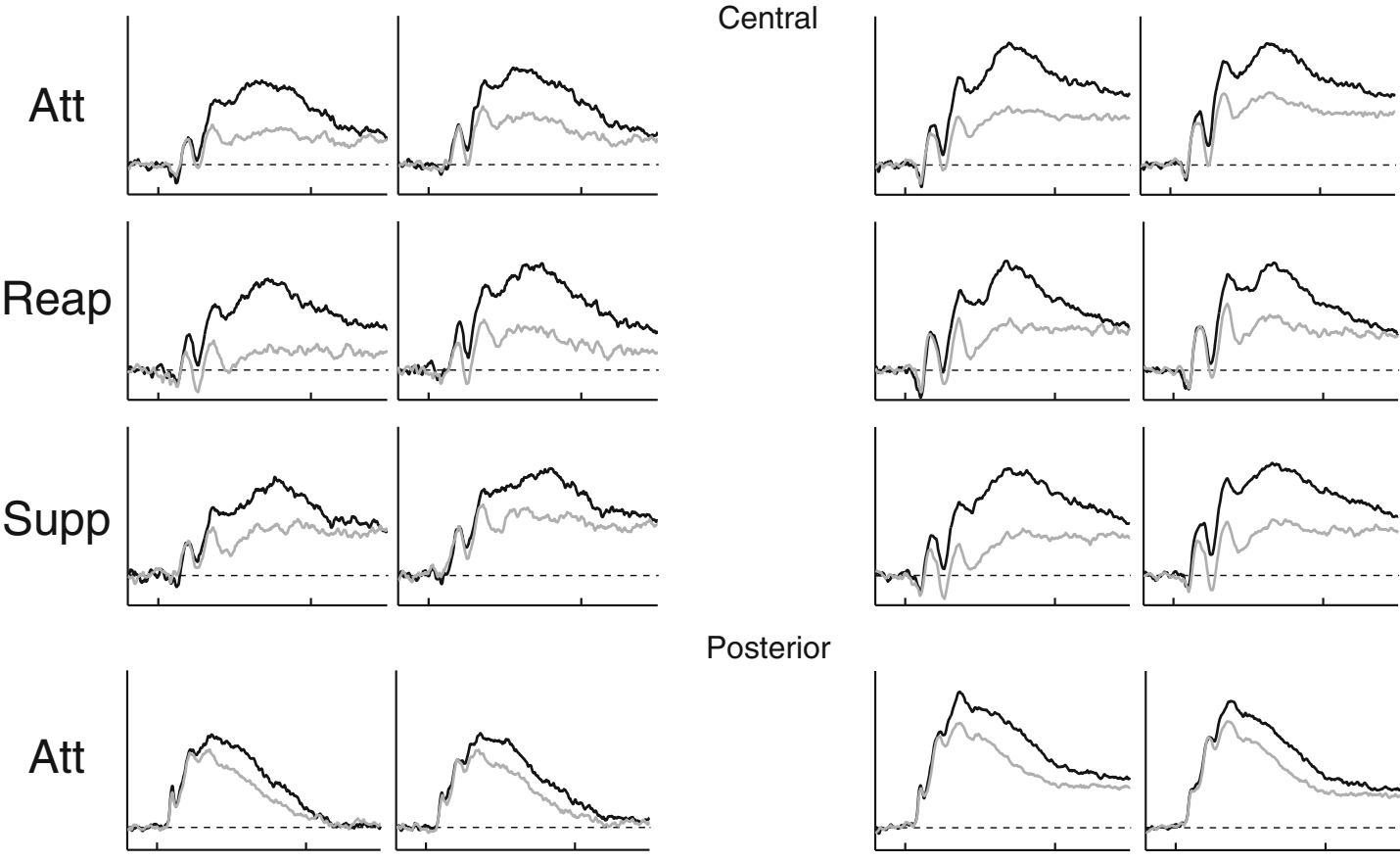

Posterior
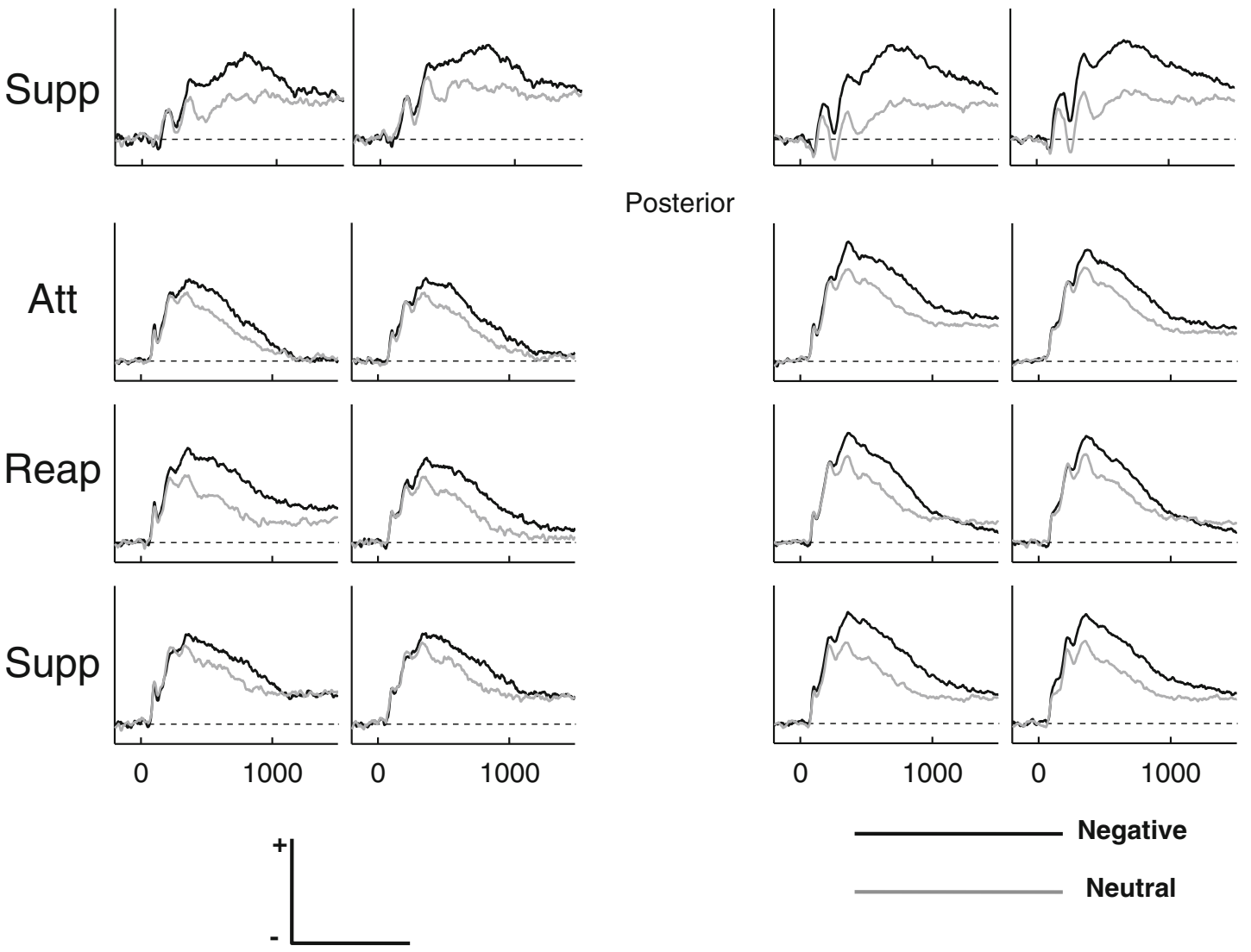

Negative

Neutral 
Fig. 1 ERPs for negative and neutral stimuli by regulation strategy and by TAS group. Waveforms are an average of electrodes in leftright frontal, central, and posterior clusters. Amplitude in microvolts $(\mu \mathrm{V})$ is on the $y$ axis, and time is on the $x$ axis

between emotional and neutral pictures during suppression, but not during reappraisal or attend conditions. In addition, these results indicate that this pattern of findings is driven by the DIF subscale of the TAS- 20 .

\section{ANOVAs}

As was explained in the Method section, we ran separate TAS $\times$ regulation $\times$ image $\times$ anterior-posterior $\times$ hemisphere ANOVAs for each time window. No reliable effects were found for the P1/N1 complex; therefore, we describe below only effects starting at the $\mathrm{P} 2$ time window (see Fig. 2).

For the $\mathrm{P} 2$ component, we found a significant main effect of image, $F(1,44)=16.43, p<.001$, with negative images showing, overall, a greater positivity than did neutral images. An anterior-posterior $\times$ image interaction was also found, $F(2,88)=5.07, p<.05$, reflecting a larger effect of image in frontal and central than in posterior sites, $F(1,44)=19.83$, $16.92,4.80, p<.001, p<.001, p<.05$, respectively. More important, a significant regulation $\times$ image $\times$ TAS interaction was found, $F(2,88)=5.32, p<.01$. Subsidiary analyses indicated that there was a significant TAS $\times$ image interaction in the suppression condition only, $F(1,44)=9.24, p<.01$, reflecting an effect of image significant only for low-TAS individuals, $F(1,27)=14.83, p<.001$. This finding indicates that $\mathrm{P} 2$ amplitudes during negative, as compared with neutral, image viewing were more positive during the suppression condition exclusively for low-TAS individuals.

For the $\mathrm{N} 2$ component, a significant main effect of image was found, $F(1,44)=37.72, p<.001$, indicating that negative images had an overall greater positivity than did neutral images. An anterior-posterior $\times$ image interaction was also found, $F(2,88)=5.96, p<.01$, probably reflecting a larger effect of image in frontal and central than in posterior sites, $F(1,44)=36.50,42.26,19.10$, respectively, all $p \mathrm{~s}<.001$. Similar to P2 findings, we also found a significant regulation $\times$ image $\times$ TAS interaction, $F(2,88)=$ $4.54, p<.05$. Subsidiary analyses indicated that there was a significant TAS $\times$ image interaction in the suppression condition only, $F(1,44)=7.11, p<.01$, driven by a significant image effect in low-TAS individuals, $F(1,27)=$ $21.20, p<.001$. This finding indicates that $\mathrm{N} 2$ amplitudes during negative, as compared with neutral, image viewing were more positive (i.e., reduced $\mathrm{N} 2$ negativity) during the suppression condition exclusively for low-TAS individuals.

Finally, in order to examine the LPP, we also analyzed consecutive $200 \mathrm{~ms}$ time windows after $400 \mathrm{~ms}$ (400-600, $600-800,800-1,000,1,000-1,200,1,200-1,400)$. A robust image effect was found in all time windows (all $p \mathrm{~s}<.001$ ).
There was also a robust anterior-posterior $\times$ image interaction found across all LPP time windows (all $p s<.01$ ). During the earliest of these time windows $(400-600 \mathrm{~ms})$, there were stronger image differences in central and posterior than in frontal sites, $F(1,44)=114.20,105.82,71.44$, respectively, all $p \mathrm{~s}<.001$. This became progressively more prominent in central than in frontal and posterior sites by $600-800 \mathrm{~ms}$, $F(1,44)=142.77,92.10,99.06$, respectively, all $p$ s $<$ .001 . For the rest of the LPP time windows, there were stronger image differences in frontal and central than in posterior sites $[800-1,000 \mathrm{~ms}, F(1,44)=99.42,125.09$, 70.82 , all $p \mathrm{~s}<.001 ; 1,000-1,200 \mathrm{~ms}, F(1,44)=61.59$, $61.18,22.76$, all $p \mathrm{~s}<.001 ; 1,200-1,400 \mathrm{~ms}, F(1,44)=$ $56.64,43.16,8.26, p<.001, p<.001, p<.01$ (in respective order)]. Consistent with previous research, these findings indicate that the LPP is a slow-going wave that is initially maximal over centro-parietal sites and becomes increasingly more pronounced at anterior sites over time (Diedrich, Naumann, Maier, Becker, \& Bartussek, 1997). A significant regulation $\times$ image $\times$ TAS interaction was found only in the earliest of these time windows (400-600 ms), $F(2,88)=4.09, p<.05$. This interaction was driven by a significant TAS $\times$ image interaction exclusive to the suppression condition, $F(1,44)=4.36, p<.05$. Subsidiary analyses revealed that the effect of image in the suppression condition was significant for both low- and high-TAS participants, although the magnitude of the effect was larger for low-TAS, $F(1,27)=36.69, p<.001$, than for high-TAS, $F(1,18)=18.80, p<.001$, individuals.

\section{Discussion}

The main finding of this study is that emotion-related ERP activity is inversely related to alexithymia scores during attempts at suppressing emotional responses. Correlational analyses indicated that this effect can be observed during

Table 2 Pearson correlation coefficients testing the relationship between TAS scores and ERP difference scores (negative minus neutral) separately for each regulation condition in each time window

\begin{tabular}{lccc}
\hline & Attend & Reappraisal & Suppression \\
\hline P1 & .01 & .07 & -.29 \\
N1 & .06 & .01 & $-.39^{* *}$ \\
P2 & -.12 & .07 & $-.39^{* *}$ \\
N2 & -.05 & .07 & $-.46^{* *}$ \\
$400-600$ & .07 & -.01 & $-.30^{*}$ \\
$600-800$ & -.03 & .11 & $-.30^{*}$ \\
$800-1000$ & .01 & .23 & -.25 \\
$1000-1200$ & .05 & .25 & -.22 \\
\hline
\end{tabular}

$* p<.05 ; * * p<.01 ; * * * p<.001$ 
Table 3 Pearson correlation coefficients testing the relationship between DIF, DDF, or EOT scores and the ERP difference scores (negative minus neutral) for the suppression condition

\begin{tabular}{lcccccccc}
\hline & $\mathrm{P} 1$ & $\mathrm{~N} 1$ & $\mathrm{P} 2$ & $\mathrm{~N} 2$ & $400-600$ & $600-800$ & $800-1000$ & $1000-1200$ \\
\hline DIF & $-.29^{*}$ & $-.31^{*}$ & $-.30^{*}$ & $-.41^{*}$ & $-.30^{*}$ & -.25 & -.19 & -.18 \\
DDF & .10 & .00 & .05 & .06 & .04 & .01 & .09 & .07 \\
EOT & -.02 & .05 & .04 & .07 & .12 & .24 & .22 & .11 \\
\hline
\end{tabular}

${ }^{*} p<.05 ; * * p<.01 ; * * p<.001$

both early $(<300 \mathrm{~ms})$ and late $(>300 \mathrm{~ms})$ emotion-related ERPs. ANOVAs suggested that this effect was the strongest in the P2 and N2 and at the beginning of the LPP (400-600 ms), where significant three-way TAS $\times$ regulation $\times$ image interactions were observed. These findings are consistent with the suggestion that alexithymia is linked to a greater expertise in the utilization of suppression-based regulation techniques because of their more frequent utilization by high alexithymics and less frequent utilization by low alexithymics (e.g., Franz et al., 2004; Swart et al., 2009). Finally, we did not find convincing evidence of a positive relationship between TAS scores and ERPs to emotion during reappraisal. We discuss these results in more detail below.

These findings are consistent with the notion that alexithymia determines the efficiency of suppression techniques because individuals scoring high or low in alexithymia vary regarding how frequently they utilize suppression to regulate emotions. Low alexithymics are less used to suppression and, therefore, are less likely to possess developed prior schemata to facilitate the utilization of this technique. Consequently, they are more likely to draw on central cognitive resources to perform the task. Given that response-focused regulation strategies are usually difficult and demanding in cognitive resources (Richards \& Gross, 2000), low alexithymics are therefore more likely to be prone to failures in their attempts at successful utilization of suppression. In contrast, individuals scoring high in alexithymia are more frequent users of suppression and, therefore, more likely to have developed overtrained schemata of efficient suppression techniques, increasing thereby their chances of success when using suppression. This explanation is consistent with previous research indicating that alexithymia scores are linked to a more frequent utilization of suppression techniques (e.g., Dimsdale et al., 1986; Franz et al., 2004; Grabe et al., 2004; Kessler et al., 2010; Richter et al., 2006; Swart et al., 2009). This explanation is also consistent with research suggesting that long-term expertise in regulation techniques improves efficiency in controlling emotional responses (Dalgleish, Yiend, Schweizer, \& Dunn, 2009; Wegner \& Gold, 1995). This explanation also fits with research suggesting that suppression-based regulation is usually demanding in cognitive resources (Richards \& Gross, 2000). Finally, this explanation is also consistent with theoretical models suggesting that failures of suppression lead to "ironic effects" - that is, the paradoxical facilitation of processing of the to-be-suppressed information (Wegner, 1994). This phenomenon can be caused by a depletion in cognitive resources (Ellis \& Ashbrook, 1987; Eysenck, Derakshan, Santos, \& Calvo, 2007), and it can generalize to paradoxical effects of suppression in emotion regulation (Dalgleish et al., 2009).

An important finding of this study is that evidence of a relationship between alexithymia and expressive suppression was found in both early and late ERP correlates of emotion. Emotion-related ERPs in late time windows $(>300 \mathrm{~ms})$ are usually referred to as the LPP and are
Fig. 2 Scalp maps of mean amplitude differences between negative and neutral conditions plotted for the P2 and N2 components and the 400 - to 600 -ms time window in those low in TAS during the suppression condition. Maxima and minima are specific to each scalp map
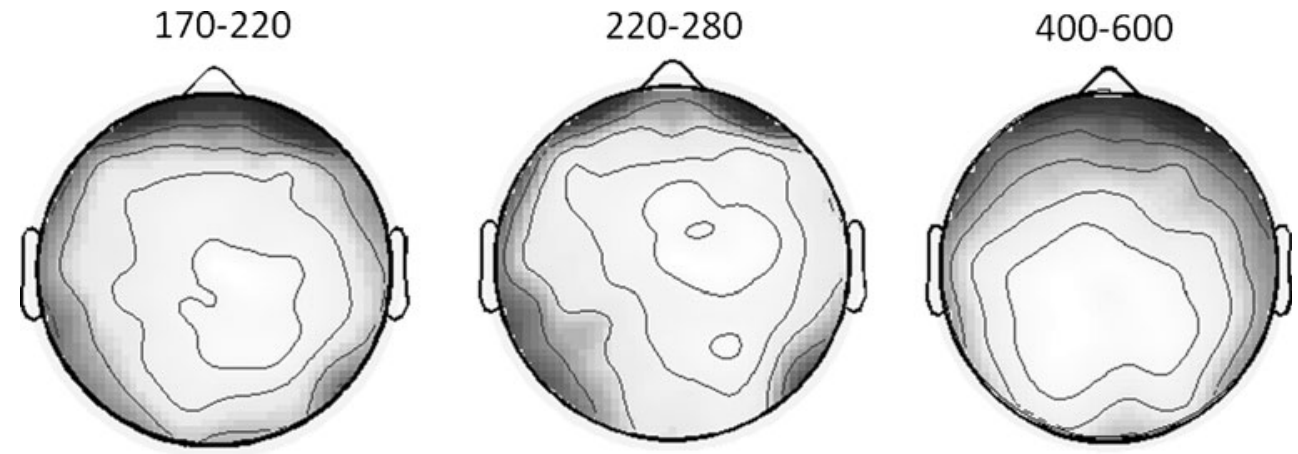

MAX 
thought to reflect overt, postperceptual emotional appraisal processes (Cuthbert et al., 2000; Schupp, Junghofer, et al., 2004), and previous research has shown that it can be reduced by effective emotion regulation (e.g., Hajcak \& Nieuwenhuis, 2006). However, the strongest effects in our study were found in early ERPs to emotion and, in particular, the P2/N2 complex. Emotion-related ERPs occurring in these time windows are thought to reflect a rapid mobilization of attention by emotionally relevant stimuli (for a review, see Olofsson et al., 2008). Furthermore, emotion-related ERPs in these time windows possess several attributes characteristic of automatic processes (Schupp et al., 2008). The present study might, therefore, suggest that alexithymia modulates the effects of suppression on early automatic processing of emotion. This explanation implies that a wilful, controlled attempt to suppress emotions can affect early automatic processes. One could argue that effects of suppression occur in later stages of the processing stream, since they rely on conscious controlled processes (Cisler \& Koster, 2010; Dunning \& Hajcak, 2009; Johnson, 2009), and therefore, effects occurring in the $\mathrm{P} 2 / \mathrm{N} 2$ latencies might be too early to be influenced by suppression. However, our regulation task used a blocked design, in which participants were in a sustained cognitive mode (suppression, reappraisal, or attend) during the entire duration of a block. Therefore, a specific regulation strategy was already in place at the onset of each picture, enabling the capture of their effects in early time windows. Furthermore, previous research has shown that "ironic" effects of suppression can be indexed by early ERP components. For instance, previous research has shown that suppressing a focus word can lead to a paradoxical increase in attention toward the word during a lexical decision task and that this can be detected as early as $260 \mathrm{~ms}$ post-stimulus-onset (Giuliano \& Wicha, 2010). Finally, previous research indicates that indices of rapid emotional processing can be influenced by ongoing cognitive tasks (King \& Schaefer, 2011; Pessoa, McKenna, Gutierrez, \& Ungerleider, 2002).

A possible alternative explanation could posit that an increased N2 negativity could reflect the implementation of inhibitory control in order to actively suppress negative emotions (Lewis et al., 2008). This would be consistent with results in the literature showing a link between the N2 and inhibitory control (Nieuwenhuis, Yeung, van den Wildenberg, \& Ridderinkhof, 2003). However, our results do not support this explanation. If N2 amplitude is related to an increase in inhibitory control involved in attempts to suppress emotions, larger N2 negativities should be observed for negative than for neutral items during suppression. In fact, $\mathrm{N} 2$ negativity was reduced, rather than increased, for emotional items, as compared with neutral items, in low-TAS individuals, and no significant difference in N2 amplitude was observed for high-TAS individuals during suppression. Another related explanation could be based on the concept of cognitive efficiency (Dennis \& Chen, 2007; Eysenck \& Calvo, 1992; Gray et al., 2005), according to which a smaller N2 negativity could reflect a more efficient utilization of cognitive control resources. Applied to our findings, it could mean that lowTAS individuals are more efficient at suppressing emotional, rather than neutral, information. However, it is unlikely and counterintuitive to assume that suppressing responses to emotionally negative stimuli would require less effort (or fewer cognitive resources) than suppressing responses to neutral pictures, which renders this explanation implausible.

A different alternative explanation suggests that the increased effect of image in the P2/N2 window might reflect an early detection of the need to suppress emotional responses. In this case, our results would reflect a better efficiency in the rapid detection of the need to suppress for low-TAS, as compared with high-TAS, individuals. This would be consistent with previous research indicating that early ERPs to the recognition of emotional faces are delayed in high alexithymics (Vermeulen, Luminet, de Sousa, \& Campanella, 2008). Although this is a potentially valid explanation, it does not fully explain our results. First, there is no a priori reason why early ERPs to emotion should be linked to the detection of the need to suppress in a specific way (rather than, for instance, the need to reappraise). If a rapid mobilization of cognitive resources to successfully suppress emotions can be adaptive, so is the need to gather resources to reappraise. This would suggest that an enhancement of early ERPs to emotion in low-TAS individuals should be observed for both regulation conditions. Yet this effect is seen only in suppression, and not in reappraisal. Second, if our results reflected a better early detection of the need to suppress in low-TAS individuals, we should expect that this would lead to a more efficient subsequent regulation. Therefore, we could predict that an enhancement of early ERPs to emotion in low-TAS individuals would be followed by a greater reduction of the LPP. However, our results show exactly the opposite pattern - that is, an inverse correlation between TAS and LPP magnitude during suppression.

A visual inspection of the waveforms suggests an apparent trend toward a positive relationship between alexithymia levels and the magnitude of ERPs to emotion during reappraisal. Yet statistical analyses did not provide convincing support for these observations. First, correlations between emotional-neutral ERP difference scores and TAS scores were nonsignificant, apart from a single positive correlation in a late time window $(1,000-1,200 \mathrm{~ms})$. Second, no significant TAS $\times$ image interaction was found in the reappraisal condition. However, we did find a significant regulation $\times$ image interaction for P2 specifically for low-TAS. Specific contrasts between negative and neutral ERPs were significant $(p<.05)$ for attend and suppression, but not in the reappraisal 
condition. In addition, the effect size of the contrasts was larger for suppression than for attend, $F=14.83$ and $F=5.58$, respectively. This result is consistent with previous research (Hajcak \& Nieuwenhuis, 2006) showing that reappraisal inhibits emotion-related ERPs. It also suggests that the emotion-related P2 is facilitated during suppression for lowTAS individuals, consistent with our overall results in the suppression condition. These findings in the reappraisal condition, added to the positive correlation found in the $1,000-1,200 \mathrm{~ms}$ window, appear to suggest that reappraisal can inhibit emotion-related ERPs for low-TAS individuals. Yet a number of important caveats need to be taken into account. First, this result is specific to the P2 component. Second, correlations between TAS and reappraisal difference scores are largely nonsignificant. Third, self-report data involving reappraisal are incongruent with ERP results in the reappraisal condition, since a negative correlation was found between TAS and self-report difference scores. A number of potential explanations are possible to account for the lack of convincing evidence of potential relationships between alexithymia and reappraisal. First, it might be possible that the effect of alexithymia on emotion regulation is mostly determined by differences in suppression, rather than reappraisal. Consistent with this explanation, Swart et al. (2009) have shown that although those high and low in alexithymia differ in how likely they are to use both suppression and reappraisal, the effect sizes of the differences between individuals scoring high and low in alexithymia were substantially larger for suppression than for reappraisal, $F=33.4$ and $F=6.8$, respectively. Second, a relationship between alexithymia and reappraisal might exist, but it might be necessary to work with a sample of high-TAS individuals with a TAS mean score approaching the extreme end of the distribution in order to obtain significant effects in reappraisal. Third, the apparent dissociation between selfreport and physiological data is not uncommon in alexithymia (Newton \& Contrada, 1994; Papciack et al., 1985; Rabavilas, 1987). Reliable self-reports of emotional responses are difficult to obtain from individuals scoring high in alexithymia, probably because emotional self-reports rely on the very abilities that are impaired in alexithymiathat is, the ability to identify and communicate feelings or emotions(Sifneos, 1973).

A number of additional questions remain open to debate. First, our findings of a correlation between TAS and suppression were not accompanied by analog results in the self-report data. This is consistent with a number of previous studies that have shown a decoupling between self-reports and physiological data in alexithymics (Newton \& Contrada, 1994; Papciack et al., 1985; Rabavilas, 1987). As was mentioned previously, this phenomenon might be related to the fact that alexithymia is linked to an alteration of the abilities on which emotional self-reports are usually reliant, such as the ability to identify and communicate feelings (Franz et al., 2004; Sifneos, 1973). Further research will be needed to investigate whether interactions between alexithymia and emotion regulation can be reflected in objective behavioral correlates of emotion (e.g., the emotional startle eyeblink; Jackson et al., 2000; Lissek et al., 2007). Second, our results show that a dimensional approach to alexithymia can lead to largely similar results as a categorical approach, since our correlational approach led to conclusions that were broadly similar to the conclusions implied by the ANOVA data. However our categorical approach had a lower statistical power because it relied on obtaining significant $F$-tests of interactions, which is a requirement that can often mask the rejection of the null hypothesis (Rosnow \& Rosenthal, 1989). Specifically, our correlational approach indicated that the relationship between TAS and emotion-related ERPs was significant in a broader range of time windows than those detected by our ANOVAs. These findings are consistent with recent evidence suggesting that alexithymia is better operationalized as a dimensional, rather than a more classical, taxometric approach (Ferguson et al., 2008; Ferguson et al., 2009; Parker et al., 2008). However, we acknowledge that the relative merits of dimensional versus taxometric approaches still need to be tested more systematically. Third, in relation to the previous point, we chose to recruit participants with a low potential for mood disorders (see the Method section), which minimizes the number of individuals in the extreme high end of the TAS distribution. As was mentioned previously, this approach was chosen in order to prevent potentially vulnerable participants from being exposed to high-arousal negative pictures. It has to be said, however, that this potential weakness should work against our hypotheses, since it minimizes the contrast between high- and low-TAS groups. Obtaining significant differences in this context suggests, therefore, that our findings are robust and do not depend on operationalizing alexithymia as a contrast between extreme ends of the alexithymia spectrum. Fourth, future research should adopt a multidimensional approach in which questionnaires such as the ERQ (Gross \& John, 2003) are explored in addition to the TAS-20, alongside questionnaires assessing major personality dimensions such as neuroticism.

Finally, an important finding of our correlational analysis is that the difficulty in identifying feelings subscale seems to account for most of the effects of the present study. This finding strengthens the viewpoint that alexithymia is not necessarily a unitary construct (Coffey, Berenbaum, \& Kerns, 2003; Gohm \& Clore, 2000; Koven \& Thomas, 2010) and that different facets of alexithymia might play specific functional roles. Previous research has divided alexithymia facets into emotional clarity (DIF + DDF) and attention to emotion (EOT), and these two dimensions have 
been associated to differences in cognitive processing (Coffey et al., 2003; Koven \& Thomas, 2010). The present findings are partially consistent with this view with regard to the differences between DIF and EOT. However, we also observed differences between DIF and DDF, which suggests that individual differences in the difficulty in identifying feelings plays a unique role in emotion regulation above and beyond the capacity to describe and communicate any feelings. Future research will be needed to confirm these findings and explore these questions further. In particular, a future avenue of research should explore whether individual differences in the DIF facet play a unique role in emotion regulation and in cognitive efficiency.

In conclusion, the results of this study suggest that alexithymia modulates both early and late emotion-related ERPs during attempts to suppress emotions. Specifically, alexithymia scores are inversely related to the magnitude of emotion-related ERP activity during suppression. These findings argue for the need to take into account affective individual differences when investigating emotion regulation processes.

Acknowledgements A. Schaefer is supported by the U.K. Biotechnology and Biological Sciences Research Council (BBSRC). The authors would like to thank Karrie Alexander for her help with data collection.

\section{References}

Bagby, R. M., Parker, J. D., \& Taylor, G. J. (1994). The twenty-item Toronto Alexithymia Scale: I. Item selection and cross-validation of the factor structure. Journal of Psychosomatic Research, 38, 23-32.

Bagby, R. M., Taylor, G. J., \& Parker, J. D. (1994). The twenty-item Toronto Alexithymia Scale: II. Convergent, discriminant, and concurrent validity. Journal of Psychosomatic Research, 38, 33-40.

Beck, A. T., \& Steer, R. A. (1987). Beck Depression Inventory Manual. San Antonio: The Psychological Corporation.

Beck, A. T., Ward, C. H., Mendelson, M., Mock, J., \& Erbaugh, J. (1961). An inventory for measuring depression. Archives of General Psychiatry, 4, 561-571.

Berg, P., \& Scherg, M. (1994). A multiple source approach to the correction of eye artifacts. Electroencephalography and Clinical Neurophysiology, 90, 229-241.

Bryant, R. A., Moulds, M., \& Guthrie, R. M. (2001). Cognitive strategies and the resolution of acute stress disorder. Journal of Traumatic Stress, 14, 213-219.

Campbell-Sills, L., Barlow, D. H., Brown, T. A., \& Hofmann, S. G. (2006a). Acceptability and suppression of negative emotion in anxiety and mood disorders. Emotion, 6, 587-595.

Campbell-Sills, L., Barlow, D. H., Brown, T. A., \& Hofmann, S. G. (2006b). Effects of suppression and acceptance on emotional responses of individuals with anxiety and mood disorders. Behaviour Research and Therapy, 44, 1251-1263.

Carretié, L., Hinojosa, J. A., Martin-Loeches, M., Mercado, F., \& Tapia, M. (2004). Automatic attention to emotional stimuli: Neural correlates. Human Brain Mapping, 22, 290-299.

Cisler, J. M., \& Koster, E. H. W. (2010). Mechanisms of attentional biases towards threat in anxiety disorders: An integrative review. Clinical Psychology Review, 30, 203-216.
Coffey, E., Berenbaum, H., \& Kerns, J. G. (2003). The dimensions of emotional intelligence, alexithymia, and mood awareness: Associations with personality and performance on an emotional stroop task. Cognition and Emotion, 17, 671-679.

Cuthbert, B. N., Schupp, H. T., Bradley, M. M., Birbaumer, N., \& Lang, P. J. (2000). Brain potentials in affective picture processing: Covariation with autonomic arousal and affective report. Biological Psychology, 52, 95-111.

Dalgleish, T., Yiend, J., Schweizer, S., \& Dunn, B. D. (2009). Ironic effects of emotion suppression when recounting distressing memories. Emotion, 9, 744-749.

Dennis, T. A., \& Chen, C. (2007). Emotional face processing and attention performance in three domains: Neurophysiological mechanisms and moderating effects of trait anxiety. International Journal of Psychophysiology, 65, 10-19.

Diedrich, O., Naumann, E., Maier, S., Becker, G., \& Bartussek, D. (1997). A frontal positive slow wave in the ERP associated with emotional slides. Journal of Psychophysiology, 11, 71-84.

Dimsdale, J. E., Pierce, C., Schoenfeld, D., Brown, A., Zusman, R., \& Graham, R. (1986). Suppressed anger and blood pressure: The effects of race, sex, social class, obesity, and age. Psychosomatic Medicine, 48, 430-436.

Dunning, J. P., \& Hajcak, G. (2009). See no evil: Directing visual attention within unpleasant images modulates the electrocortical response. Psychophysiology, 46, 28-33.

Ehring, T., Tuschen-Caffier, B., Schnulle, J., Fischer, S., \& Gross, J. J. (2010). Emotion regulation and vulnerability to depression: Spontaneous versus instructed use of emotion suppression and reappraisal. Emotion, 10, 563-572.

Ellis, H. C., \& Ashbrook, P. W. (1987). Resource allocation model of the effects of depressed mood on memory. In K. Fiedler \& J. P. Forgas (Eds.), Affect, cognition, and social behavior (pp. 25-43). New York: Hogrefe.

Eysenck, M. W., \& Calvo, M. G. (1992). Anxiety and performance: The processing efficiency theory. Cognition \& Emotion, 6, 409-434.

Eysenck, M. W., Derakshan, N., Santos, R., \& Calvo, M. G. (2007). Anxiety and cognitive performance: Attentional control theory. Emotion, 7, 336-353.

Ferguson, E., Wiliams, L., O'Connor, R., Bibby, P., \& O'Carroll, R. (2008). How should personality be used as a diagnostic risk factor for physical and psychosomatic health? A taxometric analysis of type D personality, alexithymia and hypochondriasis [Abstract]. Psychology \& Health, 23, 118.

Ferguson, E., Williams, L., O'Connor, R. C., Howard, S., Hughes, B. M., Johnston, D. W., et al. (2009). A taxometric analysis of typeD personality. Psychosomatic Medicine, 71, 981-986.

Franz, M., Schaefer, R., \& Schneider, C. (2003). Psychophysiological response patterns of high and low alexithymics under mental and emotional load conditions. Journal of Psychophysiology, 17, 203-213.

Franz, M., Schaefer, R., Schneider, C., Sitte, W., \& Bachor, J. (2004). Visual event-related potentials in subjects with alexithymia: Modified processing of emotional aversive information? The American Journal of Psychiatry, 161, 728-735.

Garnefski, N., Teerds, J., Kraaij, V., Legerstee, J., \& van den Kommer, T. (2004). Cognitive emotion regulation strategies and depressive symptoms: Differences between males and females. Personality and Individual Differences, 36, 267-276.

Giuliano, R. J., \& Wicha, N. Y. Y. (2010). Why the white bear is still there: Electrophysiological evidence for ironic semantic activation during thought suppression. Brain Research, 1316, 62-74.

Gohm, C. L., \& Clore, G. L. (2000). Individual differences in emotional experience: Mapping available scales to processes. Personality and Social Psychology Bulletin, 26, 679-697.

Grabe, H. J., Moller, B., Willert, C., Spitzer, C., Rizos, T., \& Freyberger, H. J. (2004). Interhemispheric transfer in alexithy- 
mia: A transcallosal inhibition study. Psychotherapy and Psychosomatics, 73, 117-123.

Gray, J. R., Burgess, G. C., Schaefer, A., Yarkoni, T., Larsen, R. J., \& Braver, T. S. (2005). Affective personality differences in neural processing efficiency confirmed using fMRI. Cognitive, Affective, \& Behavioral Neuroscience, 5, 182-190.

Gross, J. J. (1998a). Antecedent- and response-focused emotion regulation: Divergent consequences for experience, expression, and physiology. Journal of Personality and Social Psychology, $74,224-237$

Gross, J. J. (1998b). The emerging field of emotion regulation: An integrative review. Review of General Psychology, 2, 271-299.

Gross, J. J., \& John, O. P. (2003). Individual differences in two emotion regulation processes: Implications for affect, relationships, and well-being. Journal of Personality and Social Psychology, 85, 348-362.

Gross, J. J., \& Levenson, R. W. (1993). Emotional suppression: Physiology, self-report, and expressive behavior. Journal of Personality and Social Psychology, 64, 970-986.

Gross, J. J., \& Levenson, R. W. (1997). Hiding feelings: The acute effects of inhibiting negative and positive emotion. Journal of Abnormal Psychology, 106, 95-103.

Hajcak, G., Moser, J. S., \& Simons, R. F. (2006). Attending to affect: Appraisal strategies modulate the electrocortical response to arousing pictures. Emotion, 6, 517-522.

Hajcak, G., \& Nieuwenhuis, S. (2006). Reappraisal modulates the electrocortical response to unpleasant pictures. Cognitive, Affective, \& Behavioral Neuroscience, 6, 291-297.

Hajcak, G., \& Olvet, D. M. (2008). The persistence of attention to emotion: Brain potentials during and after picture presentation. Emotion, 8, 250-255.

Hayes, S. C., Strosahl, K., Wilson, K. G., Bissett, R. T., Pistorello, J., Toarmino, D., et al. (2004). Measuring experiential avoidance: A preliminary test of a working model. Psychological Record, 54, 553-578.

Herrmann, M. J., Huter, T., Plichta, M. M., Ehlis, A. C., Alpers, G. W., Muhlberger, A., et al. (2008). Enhancement of activity of the primary visual cortex during processing of emotional stimuli as measured with event-related functional near-infrared spectroscopy and event-related potentials. Human Brain Mapping, 29, 28 35.

Jackson, D. C., Malmstadt, J. R., Larson, C. L., \& Davidson, R. J. (2000). Suppression and enhancement of emotional responses to unpleasant pictures. Psychophysiology, 37, 515-522.

Johnson, D. R. (2009). Goal-directed attentional deployment to emotional faces and individual differences in emotional regulation. Journal of Research in Personality, 43, 8-13.

Junghofer, M., Bradley, M. M., Elbert, T. R., \& Lang, P. J. (2001). Fleeting images: A new look at early emotion discrimination. Psychophysiology, 38, 175-178.

Kashdan, T. B., Barrios, V., Forsyth, J. P., \& Steger, M. F. (2006). Experiential avoidance as a generalized psychological vulnerability: Comparisons with coping and emotion regulation strategies. Behaviour Research and Therapy, 44, 1301-1320.

Kessler, H., Kammerer, M., Hoffmann, H., \& Traue, H. C. (2010). Regulation of emotions and alexithymia: A correlative study. Psychotherapie, Psychosomatik, Medizinische Psychologie, 60, 169-174.

King, R., \& Schaefer, A. (2011). The emotional startle effect is disrupted by a concurrent working memory task. Psychophysiology, 48, 269-272.

Koven, N. S., \& Thomas, W. (2010). Mapping facets of alexithymia to executive dysfunction in daily life. Personality and Individual Differences, 49, 24-28.

Lang, P. J., Bradley, M. M., \& Cuthbert, B. N. (1999). International Affective Picture System (IAPS): Instruction manual and affective
ratings(Tech. Rep. A-4). Gainesville: University of Florida, Center for Research in Psychophysiology.

Lewis, M. D., Granic, I., Lamm, C., Zelazo, P. D., Stieben, J., Todd, R. M., et al. (2008). Changes in the neural bases of emotion regulation associated with clinical improvement in children with behavior problems. Development and Psychopathology, 20, 913 939.

Lissek, S., Orme, K., McDowell, D. J., Johnson, L. L., Luckenbaugh, D. A., Baas, J. M., et al. (2007). Emotion regulation and potentiated startle across affective picture and threat-of-shock paradigms. Biological Psychology, 76, 124-133.

Loas, G., Corcos, M., Stephan, P., Pellet, J., Bizouard, P., Venisse, J. L., et al. (2001). Factorial structure of the 20 -item Toronto Alexithymia Scale: Confirmatory factorial analyses in nonclinical and clinical samples. Journal of Psychosomatic Research, 50, $255-261$

Meng, X. L., Rosenthal, R., \& Rubin, D. B. (1992). Comparing correlated correlation coefficients. Psychological Bulletin, 111, $172-175$.

Moser, J. S., Most, S. B., \& Simons, R. F. (2010). Increasing negative emotions by reappraisal enhances subsequent cognitive control: A combined behavioral and electrophysiological study. Cognitive, Affective, \& Behavioral Neuroscience, 10, 195-207.

Newton, T. L., \& Contrada, R. J. (1994). Alexithymia and repression: Contrasting emotion-focused coping styles. Psychosomatic Medicine, 56, 457-462.

Nieuwenhuis, S., Yeung, N., van den Wildenberg, W., \& Ridderinkhof, K. R. (2003). Electrophysiological correlates of anterior cingulate function in a go/no-go task: Effects of response conflict and trial type frequency. Cognitive, Affective, \& Behavioral Neuroscience, 3 , $17-26$.

Olofsson, J. K., Nordin, S., Sequeira, H., \& Polich, J. (2008). Affective picture processing: An integrative review of ERP findings. Biological Psychology, 77, 247-265.

Olofsson, J. K., \& Polich, J. (2007). Affective visual event-related potentials: Arousal, repetition, and time-on-task. Biological Psychology, 75, 101-108.

Papciack, A. S., Feuerstein, M., \& Siegel, J. A. (1985). Stress reactivity in alexithymia: Decoupling of physiological and cognitive responses. Journal of Human Stress, 11, 135-142.

Parker, J. D. A., Keefer, K. V., Taylor, G. J., \& Bagby, R. M. (2008). Latent structure of the alexithymia construct: A taxometric investigation. Psychological Assessment, 20, 385-396.

Pastor, M. C., Bradley, M. M., Low, A., Versace, F., Molto, J., \& Lang, P. J. (2008). Affective picture perception: Emotion, context, and the late positive potential. Brain Research, 1189, $145-151$

Pessoa, L., McKenna, M., Gutierrez, E., \& Ungerleider, L. G. (2002). Neural processing of emotional faces requires attention. Proceedings of the National Academy of Sciences, 99, 11458-11463.

Rabavilas, A. D. (1987). Electrodermal activity in low and high alexithymia neurotic patients. Psychotherapy and Psychosomatics, 47, 101-104.

Richards, J. M., \& Gross, J. J. (2000). Emotion regulation and memory: The cognitive costs of keeping one's cool. Journal of Personality and Social Psychology, 79, 410-424.

Richter, J., Moller, B., Spitzer, C., Letzel, S., Bartols, S., Barnow, S., et al. (2006). Transcallosal inhibition in patients with and without alexithymia. Neuropsychobiology, 53, 101-107.

Rosnow, R. L., \& Rosenthal, R. (1989). Statistical procedures and the justification of knowledge in psychological science. The American Psychologist, 44, 1276-1284.

Schupp, H. T., Cuthbert, B. N., Bradley, M. M., Cacioppo, J. T., Ito, T., \& Lang, P. J. (2000). Affective picture processing: The late positive potential is modulated by motivational relevance. Psychophysiology, 37, 257-261. 
Schupp, H. T., Cuthbert, B. N., Bradley, M. M., Hillman, C. H., Hamm, A. O., \& Lang, P. J. (2004). Brain processes in emotional perception: Motivated attention. Cognition \& Emotion, 18, 593-611.

Schupp, H. T., Junghofer, M., Weike, A. I., \& Hamm, A. O. (2003). Attention and emotion: An ERP analysis of facilitated emotional stimulus processing. Neuroreport, 14, 1107-1110.

Schupp, H. T., Junghofer, M., Weike, A. I., \& Hamm, A. O. (2004). The selective processing of briefly presented affective pictures: An ERP analysis. Psychophysiology, 41, 441-449.

Schupp, H. T., Stockburger, J., Bublatzky, F., Junghofer, M., Weike, A. I., \& Hamm, A. O. (2008). The selective processing of emotional visual stimuli while detecting auditory targets: an ERP analysis. Brain Research, 1230, 168-176.

Sifneos, P. E. (1973). The prevalence of alexithymic characteristics in psychosomatic patients. Psychotherapy and Psychosomatics, 26, 270-285.

Swart, M., Kortekaas, R., \& Aleman, A. (2009). Dealing with feelings: Characterization of trait alexithymia on emotion regulation strategies and cognitive-emotional processing. PLoS ONE, 4, e5751.

Taylor, G. J. (2000). Recent developments in alexithymia theory and research. Canadian Journal of Psychiatry, 45, 134-142.

Taylor, G. J., Bagby, R. M., \& Parker, J. D. (1992). The Revised Toronto Alexithymia Scale: Some reliability, validity, and normative data. Psychotherapy and Psychosomatics, 57, 34-41.

Taylor, G. J., Bagby, R. M., \& Parker, J. D. A. (1997). Disorders of affect regulation: Alexithymia in medical and psychiatric illness. New York: Cambridge University Press.
Taylor, G. J., Ryan, D. P., \& Bagby, R. M. (1985). Toward the development of a new self-report alexithymia scale. Psychotherapy and Psychosomatics, 44, 191-199.

Vermeulen, N., Luminet, O., de Sousa, M. C., \& Campanella, S. (2008). Categorical perception of anger is disrupted in alexithymia: Evidence from a visual ERP study. Cognition \& Emotion, 22, 1052-1067.

Wegner, D. M. (1994). Ironic processes of mental control. Psychological Review, 101, 34-52.

Wegner, D. M., \& Gold, D. B. (1995). Fanning old flames: Emotional and cognitive effects of suppressing thoughts of a past relationship. Journal of Personality and Social Psychology, 68, 782-792.

Zech, E., Luminet, O., Rimé, B., \& Wagner, H. (1999). Alexithymia and its measurement: confirmatory factor analyses of the 20-item Toronto Alexithymia Scale and the Bermond-Vorst Alexithymia Questionnaire. European Journal of Personality, 13, 511-532.

\section{Endnote}

${ }^{1}$ This approach effectively enhances the differences in TAS scores between the high- and low-TAS groups if compared with a simple median split. However, similar results were obtained using a median split, also indicating that the TAS $\times$ image interaction was significant only for suppression in the P2/N2 complex and 400- to 600-ms time window. 